04

\title{
Динамика горения пропан-воздушной газовой смеси при ее поджиге стримерным сверхвысокочастотным разрядом
}

\author{
(ㄱ П.В. Булат,, ${ }^{1,2}$ Л.П. Грачев, ${ }^{3}$ И.И. Есаков, ${ }^{3}$ А.А. Раваев, ${ }^{3}$ Л.Г. Северинов ${ }^{3}$ \\ ${ }^{1}$ Балтийский государственный технический университет „ВОЕНМЕХ“ им. Д.Ф. Устинова, \\ 190005 Санкт-Петербург, Россия \\ ${ }^{2}$ Университет ИТМО, \\ 197101 Санкт-Петербург, Россия \\ ${ }^{3}$ ЗАО Московский радиотехнический институт РАН, \\ 117519 Москва, Россия \\ e-mail: grachev@mrtiran.ru
}

Поступило в Редакцию 14 декабря 2018г.

В окончательной редакции 14 декабря 2018 г.

Принято к публикации 15 апреля 2019г.

Описаны результаты экспериментальных исследований процесса горения пропан-воздушной газовой смеси при ее поджиге СВЧ разрядом. Смесь при атмосферном давлении с различным содержанием в ней пропана заполняет герметичную радиопрозрачную трубку, помещенную вдоль оси сфокусированного линейно поляризованного квазиоптического СВЧ пучка. Многоочаговый поджиг смеси осуществляется около одного конца трубки импульсным СВЧ разрядом с поверхностно развитой стримерной структурой. В опытах фиксируется рост газового давления в трубке по мере сгорания пропана. В них была оценена энергия СВЧ импульса, вкладываемая в высокотемпературную разрядную плазму. В опытах определен минимальный процент пропана в смеси, при котором СВЧ разряд осуществляет ее поджиг. Временной ход повышения давления в трубке по мере сгорания пропана определяет динамику процесса горения.

Ключевые слова: СВЧ пучок, стримерный разряд, пропан-воздушная смесь, мультиочаговый поджиг.

DOI: $10.21883 /$ JTF.2019.10.48168.426-18

\section{Введение}

К настоящему времени свойства газового электрического разряда, зажигаемого в квазиоптическом СВЧ пучке, достаточно подробно изучены [1-4]. Это стимулирует поиск путей практического применения такого вида разряда. Одно из возможных направлений это поджиг горючей смеси в схемах импульсных детонационных двигателей [5].

Газовый СВЧ разряд, зажигаемый в квазиоптическом электромагнитном (ЭМ) пучке с ТЕМ структурой поля в сантиметровом диапазоне частот $f \approx(1.5-30) \mathrm{GHz}$ при газовых давлениях $p$, больших $10^{4} \mathrm{~Pa}$, и длительности СВЧ импульса $\tau_{p u l}$ в десятки микросекунд, реализуется в пространственно развитой стримерной структуре. При этом при мощности пучка $P_{\text {beam }}$ масштаба нескольких киловатт или даже мегаватта электрический пробой газа должен быть инициирован, например, помещением в заданную область СВЧ пучка ЭМ вибратора [6].

В работе [6] полуволновый вибратор помещался на внутренней поверхности диэлектрической радиопрозрачной трубки „вдоль“ вектора электрической составляющей линейно поляризованной СВЧ волны $\mathbf{E}_{\mathbf{0}}$. Стримерные каналы зажигаемого при этом инициированного СВЧ разряда в воздухе атмосферного $p$, распространяясь по внутренней поверхности трубки навстречу волновому вектору $\mathbf{k}$ ЭМ пучка с максимальной скоростью масштаба нескольких единиц, умноженных на $10^{5} \mathrm{~cm} / \mathrm{s}$, в течение $\tau_{\text {pul }}=40 \mu \mathrm{s}$, могли заполнять внутреннюю поверхность трубки диаметром $d=3.6 \mathrm{~cm}$ на длину $L_{\mathrm{dis}}$ до $15 \mathrm{~cm}$. В опытах трубка могла заполняться газовой пропан-воздушной горючей смесью. При этом в зависимости от длины разрядной области $L_{\mathrm{dis}}$ реализовывался или дефлаграционный режим распространения пламени по трубке, или режим практически мгновенного многоочагового поджига смеси почти по всей длине трубки. При этом если в первом случае время сгорания пропана

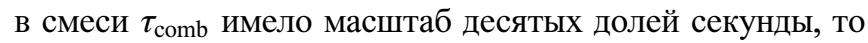

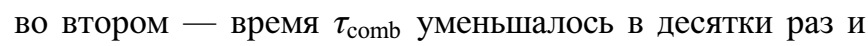
имело масштаб нескольких ms.

Описываемые в настоящей работе эксперименты выполнялись по представленной в работе [6] схеме, но в них использовалась трубка длиной $L$, существенно большей длины $L_{\mathrm{dis}}$. Трубка по-прежнему заполнялась пропан-воздушной смесью с разным содержанием в ней пропана. Энергия быстро сгорающего пропана в подожженной СВЧ разрядом смеси в прилегающей к одному концу трубки области могла стимулировать взрывной характер распространения пламени по остальной длине трубки. В опытах фиксировалось временное изменение газового давления в трубке $\Delta p$ по мере сгорания пропана. Целью экспериментов по-прежнему было определение возможности многоочагового поджига горючей смеси инициированным поверхностным стримерным СВЧ разрядом и определение нижней границы поджига по содержанию в смеси пропана. Кроме того, наличие в 
аппаратуре измерителя $\Delta p$ позволило оценить энергию СВЧ импульса, вкладываемую в высокотемпературную разрядную плазму, и измерить временной темп сгорания пропана в смеси $\Delta p / \Delta t$ при разном процентном содержании в ней пропана.

\section{Экспериментальные условия}

Эксперименты выполнялись на описанной в работе [6] установке. Ее схема приведена на рис. 1.

В опытах использовались одиночные СВЧ импульсы мощностью $P_{\text {beam }}=600 \mathrm{~kW}$, длительностью $\tau_{\text {pul }}=40 \mu \mathrm{s}$ и длиной волны СВЧ излучения $\lambda=8.9 \mathrm{~cm}$. В установке сформированный линейно поляризованный ЭМ пучок с характерным поперечным диаметром в $60 \mathrm{~cm}$ излучается в СВЧ безэховую рабочую камеру. Пучок падает на сферически вогнутое металлическое зеркало и, отразившись от него, фокусируется. Поперечная фокусная плоскость пучка отстоит от среза зеркала на расстоянии $x_{f}=10.5 \mathrm{~cm}$. Характерные поперечные размеры пучка в его фокусной области равны нескольким сантиметрам.

В опытах, как показано на рис. 1, вдоль оси ЭМ пучка может помещаться радиопрозрачная акриловая рабочая трубка внутренним диаметром $d=34 \mathrm{~mm}$ и наружным $-40 \mathrm{~mm}$. Торцы трубки герметизированы диэлектрическими капролоновыми пробками. При этом длина трубки между их внутренними торцами $L=43 \mathrm{~cm}$. По оси ближней к зеркалу пробки с наружной еe стороны подключен предохранительный газовый клапан. Он при определенной величине давления $p$ в трубке начинает выпускать из нее газ. К дальней от зеркала пробке подключены две вспомогательные резиновые трубки длиной примерно равной $1 \mathrm{~m}$, наружным диаметром $8 \mathrm{~mm}$ и внутренним $-3 \mathrm{~mm}$.

К одной из этих трубок (нижней на схеме) подключен статический измеритель газового давления. К ней же подключены тракты подвода к рабочей трубке воздуха и пропана $\mathrm{C}_{3} \mathrm{H}_{8}$. Эта система имеет соответствующие клапаны: K1, K2 и К3. В опытах рабочая трубка первоначально откачивается до $p_{0} \approx 3 \cdot 10^{2} \mathrm{~Pa}$. Затем она

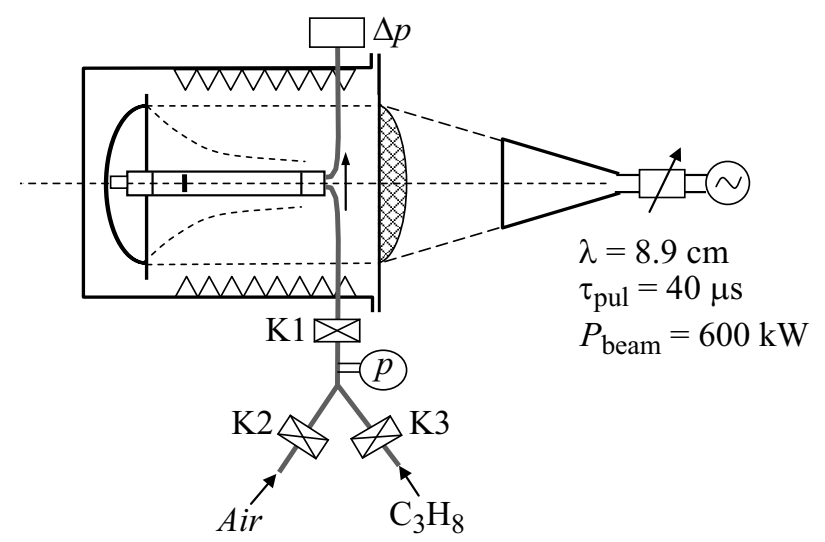

Рис. 1. Схема экспериментальной установки.

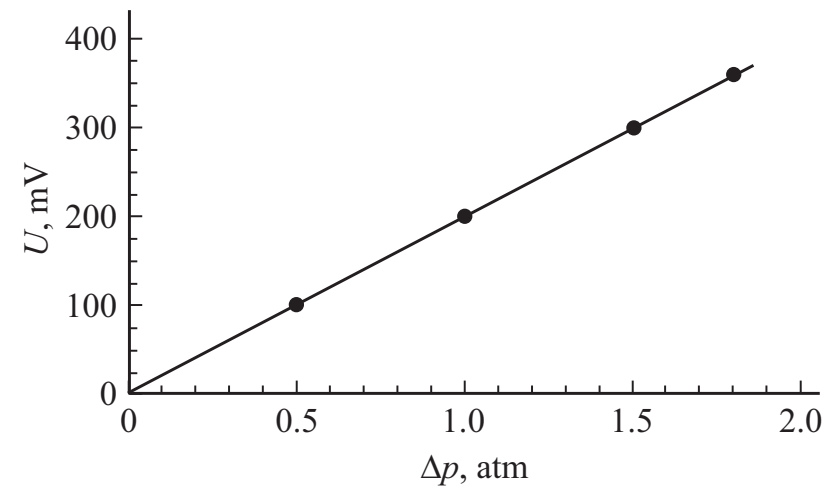

Рис. 2. Калибровочные данные динамического измерителя давления.

заполняется пропаном до заданного давления $p_{0}+p_{\text {prop }}$ и при закрытом клапане К3 дозаполняется воздухом до атмосферного давления. Для того чтобы воздух и пропан в рабочей трубке смешались, в опытах после ее заполнения газами при закрытом клапане К1 делается пауза в течение времени, не меньшем $4 \mathrm{~min}$.

К верхней на схеме вспомогательной трубке подключен динамический измеритель давления в рабочей трубке. Он выполнен на основе датчика 24РССFА6Д, который имеет паспортное время задержки $5 \mathrm{~ms}$. Сигнал с датчика подается на осциллограф. На рис. 2 приведена экспериментально определенная калибровочная зависимость напряжения $U$ с динамического датчика от превышения давления в рабочей трубке $\Delta p$ по отношению к атмосферному давлению. Из нее следует, что чувствительность динамического датчика $S=1 / 200$, $0.1 \mathrm{MPa} / \mathrm{mV}$.

В процессе калибровки измерителя $\Delta p$ выяснилось, что предохранительный клапан рабочей трубки начинает выпускать из нее воздух при избыточном давлении $\Delta p \approx 0.18 \mathrm{MPa}$ и практически не допускает повышения статического давления в рабочей трубке, большего $\Delta_{p}=0.21 \mathrm{MPa}$.

Величина $\Delta p=0.18 \mathrm{MPa}$ по рис. 2 указывает, что в процессе анализа результатов опытов при превышении показаний динамического измерителя давления значения $U_{\text {thres }}=350 \mathrm{mV}$ надо учитывать наличие в схеме установки предохранительного клапана. Это значение $\Delta p$ по формуле

$$
\Delta p=n \cdot k \cdot \Delta T ; \quad \mathrm{N} / \mathrm{m}^{2},
$$

(где $n ; 1 / m^{3}-$ концентрация молекул газа, а $k=1.38 \cdot 10^{-23} \mathrm{~J} / \mathrm{K}-$ постоянная Больцмана, может указать и граничное значение измеряемого увеличения температуры газа $\Delta T$ в рабочей трубке при выделении в ней тепловой энергии. Для воздуха атмосферного давления $n=2.57 \cdot 10^{25} 1 / \mathrm{m}^{3}$ и по (1) при $\Delta p=0.18 \mathrm{MPa}=1.8 \cdot 10^{5} \mathrm{~N} / \mathrm{m}^{2}$ граничное $\Delta T=500 \mathrm{~K}$. Для справки отметим, что это значение $\Delta T$ практически равно температуре воспламенения пропан-воздушной горючей смеси [7]. 


\section{СВЧ разряд}

В экспериментах разряд инициировался ЭМ вибратором. Он был выполнен из медной проволоки диаметром $1 \mathrm{~mm}$ и длиной $50 \mathrm{~mm}$. Проволока приклеивалась к внутренней боковой поверхности трубки „вдоль“ вектора $\mathbf{E}_{\mathbf{0}}$ СВЧ пучка. Расстояние от ЭМ вибратора до среза, фокусирующего ЭМ волну зеркала, равнялось $11 \mathrm{~cm} . \mathrm{Ha}$ рис. 3 приведена фотография, иллюстрирующая горящий СВЧ разряд.

На фотографии слева находится вогнутая поверхность фокусирующего зеркала. СВЧ разряд горит около левого торца рабочей трубки, прилегая к заглушающей ее пробке. Разряд представляет собой плазменные стримерные каналы, горящие по внутренней поверхности трубки [6]. Масштабом изображения на фотографии может служить диаметр трубки и, следовательно, по рис. 3 длина разрядной области $L_{d i s} \approx 15 \mathrm{~cm}$. С правой стороны трубки сравнительно ярко светится внутренний торец заглушающей ее дальней от фокусирующего зеркала пробки. Остальные элементы на фотографии являются конструктивными.

На рис. 4 приведены осциллограммы с датчика $\Delta p$. На них вертикальный масштаб $-20 \mathrm{mV}$ на большое деление, а горизонтальный $-5 \mathrm{~ms}$ на большое деление. Верхняя осциллограмма иллюстрирует уровень пятидесятигерцовой наводки на датчик. Она получена без включения СВЧ импульса. Включено только питание электронной схемы датчика. Нижняя осциллограмма снята уже при горении СВЧ разряда. На ней задержка включения СВЧ импульса относительно начала развертки луча осциллографа равна $2.5 \mathrm{~ms}$, задержка реагирования датчика за счет длины вспомогательной трубки, подводящей к нему сигнал от рабочей трубки, равна примерно $3 \mathrm{~ms}$ и задержка реагирования самого датчика $-5 \mathrm{~ms}$. С учетом этих времен уровень сигнала за счет теплового повышения давления воздуха в рабочей трубке при горении СВЧ разряда начинает расти только через $t_{d e l}=10.5 \mathrm{~ms}$. При этом за вычетом амплитуды наводки максимальный рост сигнала $\Delta U=12 \mathrm{mV}$. С уче-

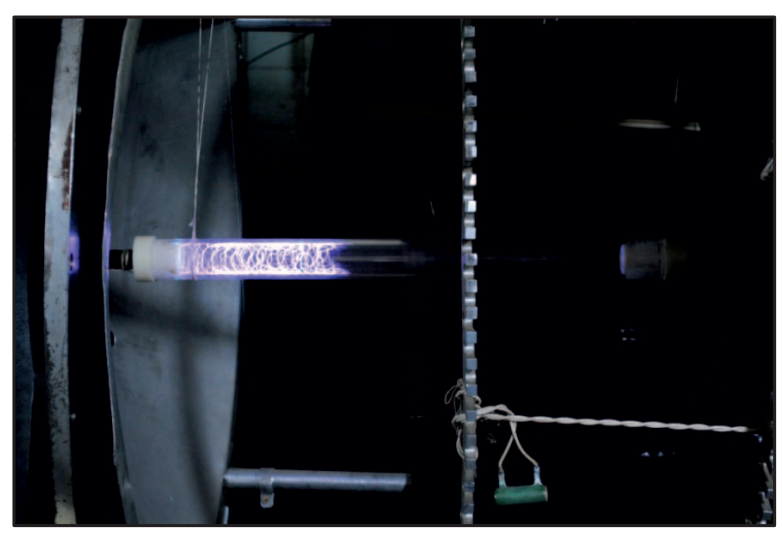

Рис. 3. Внешний вид СВЧ разряда.

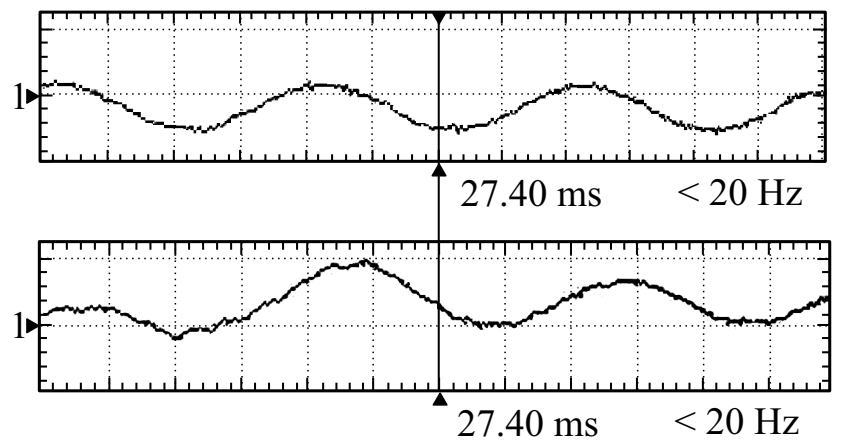

Рис. 4. Показания динамического датчика давления при горении только разряда.

том определенной чувствительности $\mathrm{S}$ датчика этому значению $\Delta U$ соответствует рост давления в трубке $\Delta p=\Delta U \cdot S=6 \cdot 10^{3} \mathrm{~N} / \mathrm{m}^{2}$. По (1) этому значению $\Delta p$ соответствует повышение температуры воздуха в трубке $\Delta T=17 \mathrm{~K}$.

Для расчета энергии $W$, требующейся для нагрева воздуха на такую величину $\Delta T$, можно воспользоваться формулой

$$
W=c_{v} \cdot M_{\text {air }} \cdot \Delta T,
$$

где теплоемкость воздуха при постоянном объеме $c_{V}=7.2 \cdot 10^{2} \mathrm{~J} /(\mathrm{kg} \cdot \mathrm{K})$, а $M_{\text {air }}$ - масса нагреваемого воздуха. В рассматриваемом случае $M_{\text {air }}=\rho_{\text {air }} \cdot V_{p}=$ $=4.8 \cdot 10^{-4} \mathrm{~kg}$ при плотности воздуха при атмосферном давлении $\rho_{\text {air }}=1.23 \mathrm{~kg} / \mathrm{m}^{3}$ и объеме трубки $V_{p}=3.9 \cdot 10^{-4} \mathrm{~m}^{3}$. Следовательно, $W=6 \mathrm{~J}$.

При энергии СВЧ импульса $W_{\mathrm{MW}}=P_{\text {beam }} \cdot \tau_{\text {pul }}=24 \mathrm{~J}$ отношение $W / W_{\mathrm{MW}}=0.25$. Следовательно, в данной экспериментальной постановке в нагрев воздуха в СВЧ разрядной плазме идет $25 \%$ энергии $W_{\mathrm{Mw}}$. По литературным данным [8] для точечного поджига пропанвоздушной смеси требуется энергия $W_{i g} \gtrsim 50 \mathrm{~mJ}$. Следовательно, энергии $W$ в рассматриваемых экспериментальных условиях будет хватать для многоочагового поджига горючей смеси при заполнении ею рабочей трубки.

Нижняя концентрационная граница зоны воспламенения пропан-воздушной смеси

Наличие в схеме динамического датчика измерения $\Delta p$ позволяет экспериментально определить нижнюю границу зоны воспламенения пропан-воздушной смеси при использовании для ее поджига СВЧ разряда по относительному содержанию пропана в смеси.

В работе [9] приведено выражение, позволяющее рассчитать стехиометрический состав пропан-воздушной смеси. Оно показывает, что для полного сгорания, например, одного g пропана требуется $16 \mathrm{~g}$ воздуха: $\eta_{s t}=1 / 16$. В рассматриваемых экспериментальных условиях могут быть измерены величины $p_{\text {prop }}$ и $p_{\text {air. }}$ Они позволяют рассчитать величину $\eta=M_{\text {prop }} / M_{\text {air }}$, где $M_{\text {prop }}$ - масса 
пропана в смеси, а $M_{\text {air }}$ - масса воздуха в ней:

$$
\eta=\frac{p_{\text {prop }}}{p_{\text {air }}} \cdot \frac{m_{a \text { prop }}}{m_{a \text { air }}},
$$

при массе молекулы пропана $m_{a \text { prop }}=8 \cdot 10^{-26} \mathrm{~kg}$, а воздуха $-m_{a \text { air }}=4.8 \cdot 10^{-26} \mathrm{~kg}$. Эта формула позволяет рассчитать стехиометрическое значение $p_{\text {prop st }}$ при экспериментальном значении $p_{\text {air }}=76 \cdot 10^{2} \mathrm{~Pa}$ : $P_{\text {prop st }}=2850 \mathrm{~Pa}$.

В работе [8] приведено объемное процентное соотношение пропана и воздуха в смеси на нижней границе зоны воспламенения данной смеси: $V_{\text {prop }} / V_{\text {air }}=2.12 \%$. Это отношение позволяет по формуле

$$
\eta=\frac{V_{\text {prop }}}{V} \cdot \frac{m_{a \text { prop }}}{m_{a \text { air }}},
$$

рассчитать граничное массовое соотношение компонентов в смеси $\eta_{\text {thres }}=0.53 / 16$ и по (3) граничное значение $p_{\text {propth }}=1.5 \cdot 10^{3} \mathrm{~Pa}$ при экспериментальной величине $p_{\text {air }}=7.6 \cdot 10^{4} \mathrm{~Pa}$.

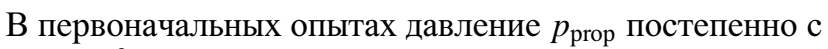
шагом $10^{2} \mathrm{~Pa}$. увеличивалось, начиная с $p_{\text {prop }}=900 \mathrm{~Pa}$. При этом до $p_{\text {prop }}=1300$ Ра при включении СВЧ импульса не менялись ни внешний вид рабочей трубки с горящим СВЧ разрядом, продолжая быть аналогичным рис. 3, ни форма осциллограммы с измерителя $\Delta p$, продолжая оставаться аналогичной нижней осциллограмме, приведенной на рис. 4. В диапазоне $p_{\text {prop }}=(14-15) \cdot 10^{2}$ Ра внешний вид рабочей трубки не менялся, а сигнал на осциллограмме резко возрастал. Затем с ростом $p_{\text {prop }}$ это возрастание становилось плавным. Диапазон $p_{\text {prop }}=(14-15) \cdot 10^{2}$ Ра практически

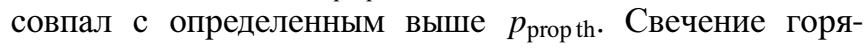
щего газа в трубке начинало наблюдаться только при $p_{\text {prop }} \geqslant 1.7 \cdot 10^{2} \mathrm{~Pa}$. Оно сначала появляется в области трубки, прилегающей к разряду. Затем с ростом $p_{\text {prop }}$ протяженность светящейся области возрастала и перекрывала всю длину трубки при $p_{\text {prop }} \geqslant \cdot 10^{3} \mathrm{~Pa}$.

В работе [8] указывается, что при значении $\eta$ лишь не намного превышающем $\eta_{\mathrm{th}}$ должно фиксироваться холодное, без свечения горение горючей смеси, причем лишь с частичным выделением энергии. Для оценки последнего утверждения на рис. 5 приведена осциллограмма с датчика $\Delta p$ при $p_{\text {prop }}=1.5 \cdot 10^{3} \mathrm{~Pa}$.

На осциллограмме вертикальный масштаб - $50 \mathrm{mV}$ на деление, горизонтальный, как и на всех последующих аналогичных осциллограммах, - $5 \mathrm{~ms}$ на деление. Треугольный значок с цифрой 1 внизу около левой вертикальной стороны поля осциллограммы показывает нулевой уровень сигнала, а аналогичный значок слева над верхней ограничивающей линией указывает момент включения СВЧ импульса.

Можно предположить, что начальный участок осциллограммы в течение времени $t \approx 5 \mathrm{~ms}$ соответствует процессу горения смеси в области трубки на длине СВЧ разряда. Следующий участок осциллограммы в течение $t \approx 8 \mathrm{~ms}$ иллюстрирует распространение процесса

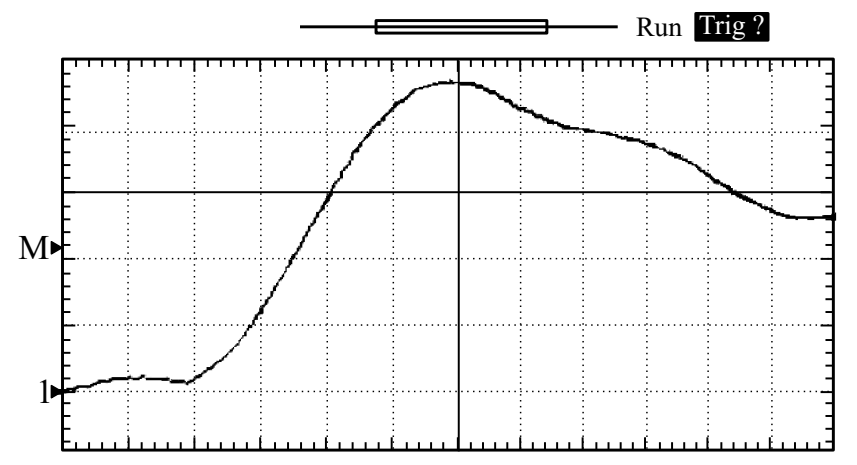

Рис. 5. Осциллограмма зависимости $\Delta p$ от $t$ при $p_{\text {prop }}=1.5 \cdot 10^{3} \mathrm{~Pa}$ (вертикальный масштаб $-50 \mathrm{mV} / \mathrm{div}$, горизонтальный $-5 \mathrm{~ms} / \mathrm{div})$.

горения вдоль остальной длины трубки. И, наконец, заключительный участок переднего фронта осциллограммы связан с этапом догорания пропана в смеси. На осциллограмме амплитуда сигнала $U_{\max }=240 \mathrm{mV}$, что соответствует $\Delta p=0.12 \mathrm{MPa}$. Для такого увеличения давления в рабочей трубке по (1) воздух в ней должен нагреться на $\Delta T=350 \mathrm{~K}$, что по (2) требует вложения в него тепловой энергии $W=120 \mathrm{~J}$. Сравним это значение энергии с энергией сгорания пропана $W_{\text {prop }}$ при его полном сгорании. При $p_{\text {prop }}=1.5 \cdot 10^{3} \mathrm{~Pa}$ масса пропана в рабочей трубке $M_{\text {prop }}=n_{\text {prop }} \cdot m_{\text {a prop }} \cdot V_{\mathrm{p}}=1.5 \cdot 10^{-5} \mathrm{~kg}$, где учтено, что при $p_{\text {prop }}=1.5 \cdot 10^{3} \mathrm{~Pa}$ концентрация его молекул $n_{\text {prop }}=2.57 \cdot 10^{25} \cdot(15 / 760)=5 \cdot 10^{23} 1 / \mathrm{m}^{3}$. Теплота сгорания пропана $Q_{\mathrm{com}}=4.64 \cdot 10^{7} \mathrm{~J} / \mathrm{kg}[8]$ и при полном сгорании рассчитанной его массы выделилась бы энергия $W_{\text {com }}=Q_{\text {com }} \cdot M_{\text {prop }}=7 \cdot 10^{2} \mathrm{~J}$. Следовательно, при таком процентном содержании пропана в его смеси с воздухом сгорает всего 17\% пропана.

Таким образом, в соответствии с [8] лишь частичное выделение энергии и отсутствие свечения и указывают на процесс холодного горения.

\section{Динамика горения}

По переднему фронту приведенной на рис. 5 осциллограммы может быть определена динамика повышения давления в рабочей трубке по мере сгорания в ней пропана $\Delta p / \Delta t$. Максимальная величина этого отношения $\Delta p / \Delta t=85 \mathrm{~atm} / \mathrm{s}$. Она выше литературного значения [10] $\Delta p / \Delta t=2 \mathrm{MPa} / \mathrm{s}$, характерного для процесса дефлаграционного горения смеси, подожженной точечным источником. В этой работе указана также величина $\Delta p / \Delta t=20 \mathrm{MPa} / \mathrm{s}$, характерная для взрывного характера горения пропан-воздушной смеси.

На рис. 6-9 приведены аналогичные рис. 5 осциллограммы при $p_{\text {prop }}=1.8,2.1,2.4$ и $2.7 \cdot 10^{3} \mathrm{~Pa}$. Над ними помещены соответствующие им фотографии рабочей трубки при поджиге горючей смеси СВЧ разрядом. 
Сравнение приведенных на этих рисунках данных показывает, что температура в трубке поднимается до температуры воспламенения пропан-воздушной смеси только при $p_{\text {prop }} \geqslant 2.1 \cdot 10^{3} \mathrm{~Pa}$ т. е. $\eta=M_{\text {prop }} / M_{\text {air }} 0.74 / 16$. При этом же значении $p_{\text {prop }}$ свечение горящей смеси начинает перекрывать всю длину трубки. С ростом в смеси pprop максимальный темп повышения давления в трубке $\Delta p / \Delta t$ плавно увеличивается от $8.5 \cdot 10^{4} \mathrm{~Pa} / \mathrm{s}$ до $14.0 \mathrm{MPa} / \mathrm{s}$. При этом он все время выше этого значения, характерного для дефлаграционного горения смеси, но меньше взрывного его характера.
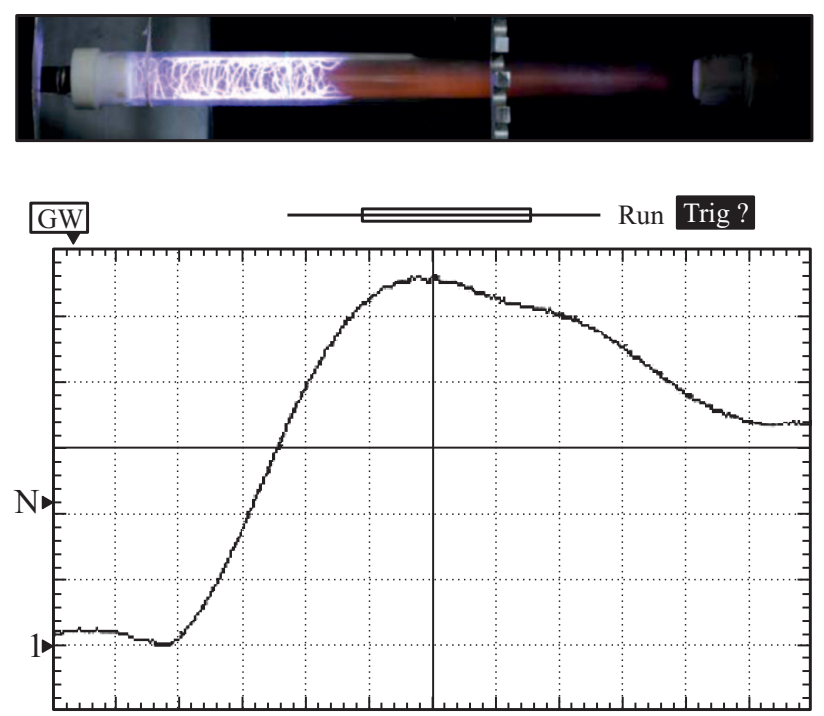

Рис. 6. Осциллограмма зависимости $\Delta p$ от $t$ при $p_{\text {prop }}=1.8 \cdot 10^{3} \mathrm{~Pa}$ (вертикальный масштаб $-50 \mathrm{mV} /$ div, горизонтальный $-5 \mathrm{~ms} / \mathrm{div})$.
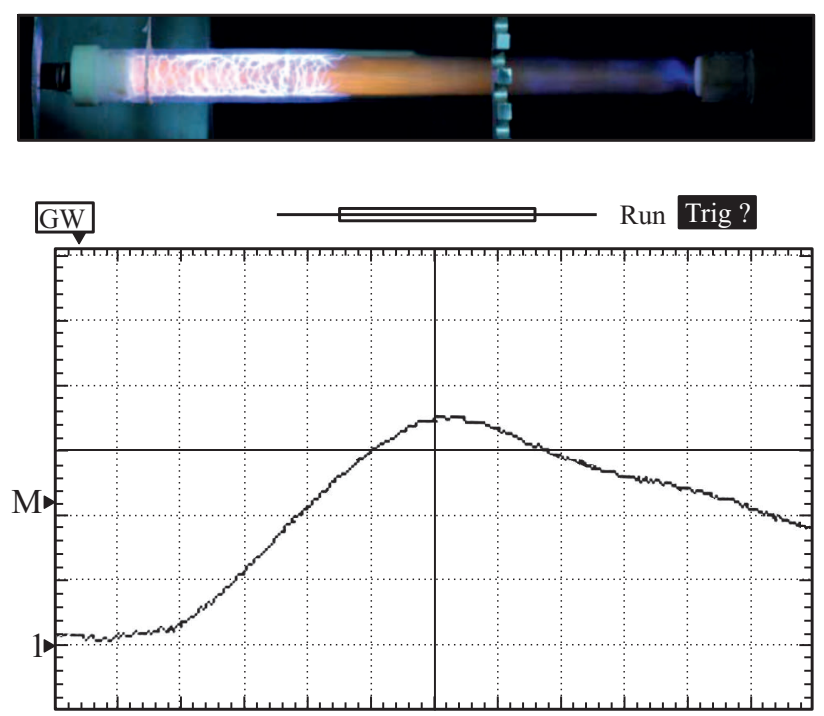

Рис. 7. Осциллограмма зависимости $\Delta p$ от $t$ при $p_{\text {prop }}=2.1 \cdot 10^{3} \mathrm{~Pa}$ (вертикальный масштаб $-100 \mathrm{mV} / \mathrm{div}$, горизонтальный $-5 \mathrm{~ms} / \mathrm{div})$.
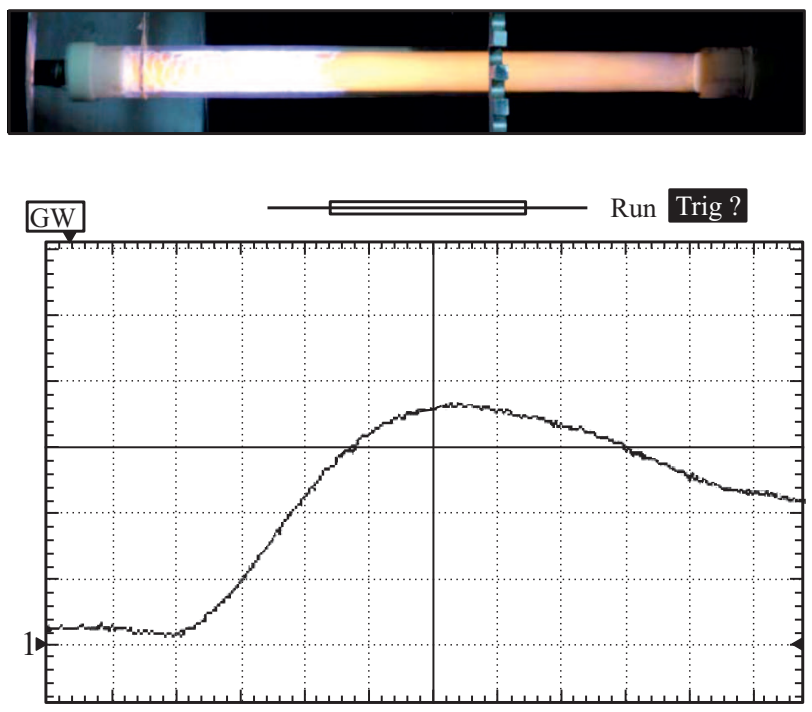

Рис. 8. Осциллограмма зависимости $\Delta_{p}$ от $t$ при $p_{\text {prop }}=2.4 \cdot 10^{3}$ Ра вертикальный масштаб $-100 \mathrm{mV} / \mathrm{div}$, горизонтальный $-5 \mathrm{~ms} / \mathrm{div})$.
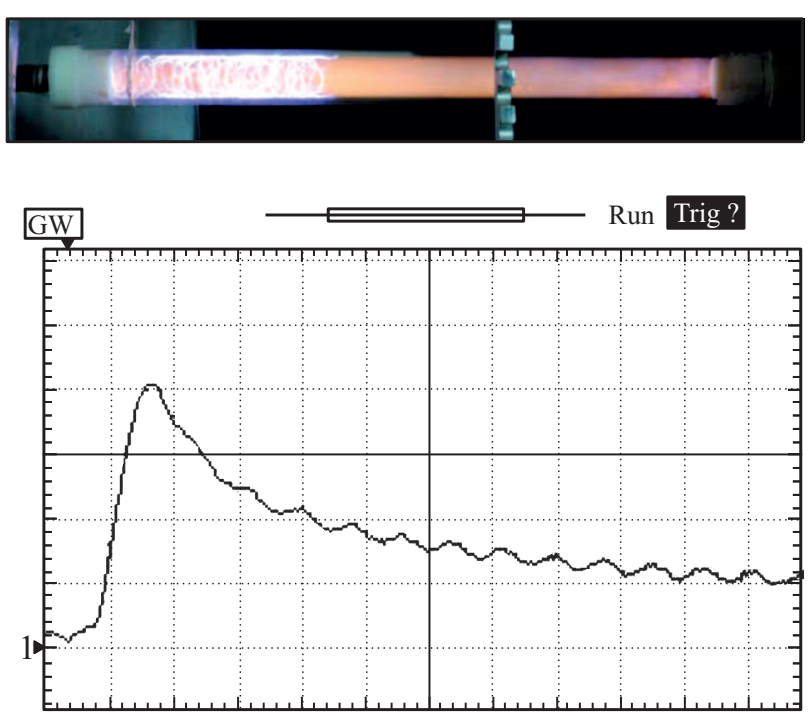

Рис. 9. Осциллограмма зависимости $\Delta p$ от $t$ при $p_{\text {prop }}=2.7 \cdot 10^{3} \mathrm{~Pa}$ (вертикальный масштаб $-100 \mathrm{mV} / \mathrm{div}$, горизонтальный $-25 \mathrm{~ms} / \mathrm{div})$.

Рис. 9 соответствует практически стехиометрическому составу смеси: $\eta=0.95 / 16$. На этом рисунке специально помещена осциллограмма при горизонтальной развертке луча осциллографа, равной $25 \mathrm{~ms} / \mathrm{div}$. Она наглядно демонстрирует, что, как и при всех значениях $\eta$, температура в трубке после завершения процесса горения падает почти за десятые доли секунды. На этой осциллограмме значение $U$ уменьшается до $100 \mathrm{mV}$, что соответствует $\Delta p=5 \cdot 10^{4} \mathrm{~Pa}$, или повышению температуры в трубке $\Delta T=140 \mathrm{~K}$. 


\section{Заключение}

Таким образом, эксперименты продемонстрировали возможность многоочагового поджига пропан-воздушной газовой смеси в диэлектрической трубке импульсным стримерным СВЧ разрядом с поверхностно-развитой плазменной стримерной структурой. В них определена нижняя граница зоны воспламенения этой смеси по массовому составу в ней пропана: $\eta \approx 0.5 / 16$. В опытах показано, что до $\eta \approx 0.63 / 16$ реализуется холодное, без свечения, горение этой смеси лишь с частичным выделением энергии. При использованном способе поджига на темп даже холодного горения влияет не только энергия высокотемпературной плазмы СВЧ разряда, но и энергия сгорания пропана в объеме трубки с длиной СВЧ разряда.

Полученные экспериментальные результаты могут быть использованы, например, при разработке импульсного детонационного двигателя.

В работе наметились и несколько невыясненных научных вопросов. Основной из них - чем объясняется быстрое охлаждение области горения по завершению процесса горения. Темп этого охлаждения не укладывается в механизм теплопроводности. Не ясна и физика перехода беспламенного горения смеси в процесс обычного ее горения.

\section{Финансирование работы}

Работа выполнена при финансовой поддержке Министерства Образования и науки Российской Федерации (соглашение № 14.577.21.0277, уникальный идентификатор проекта RFMEFI57717X0277).

\section{Конфликт интересов}

Авторы заявляют, что у них нет конфликта интересов.

\section{Список литературы}

[1] Грачев Л.П., Есаков И.И., Александров К.В., Раваев А.А., Северинов Л.Г., Ходатаев К.В. Газовый электрический разряд в квазиоптическом СВЧ пучке. М.: АО „МРТИ PAH“', 2015. $187 \mathrm{c}$.

[2] Батанов Г.М., Грицинин С.И., Коссый И.А., Магунов А.Н., Силаков В.П., Тарасова Н.М. // Тр. Физического ин-та им. П.Н. Лебедева АН СССР. 1985. Т. 160. С. 174-203.

[3] Зарин А.С., Кузовников А.А., Шибков В.М. Свободно локализованный СВЧ-разряд в воздухе. М.: Нефть и газ, 1996.

[4] Высокочастотный разряд в волновых полях. Сб. науч. тр. ИПФ АН СССР / Отв. ред. А.Г. Литвак. Горький. 1988.

[5] Bulat P., Esakov I., Volkov K. // Horizons in World Physics. 2018. Vol. 295. N 6. P. 139-190.

[6] Александров К.В., Буслеев Н.И., Грачев Л.П., Есаков И.И., Раваев А.А. // ЖТФ. 2018. Т. 88. Вып. 2. С. 174-179.
[7] Сэйитиро Кумагаи Горение. М.: Химия, 1979. 255 с.

[8] Бартльме Ф.М. Газодинамика горения. М.: Энергоиздат, 1981. $279 \mathrm{c}$.

[9] Абрамович Г.Н. Прикладная газовая динамика. М.: Мир, $1968.437 \mathrm{c}$.

[10] Маркштейн Дж.Г. Нестационарное распространение пламени. М.: Мир, 1968. 437 c. 